\title{
Exploration on Shufeng Jiedu Capsule for Treatment of COVID-19 Based on Network Pharmacology and Molecular Docking
}

\author{
Yanwu Zhao, Junhui Hu*, Jiang Song, Xinhong Zhao, Yanjing Shi, Yanping Jiang \\ Department of Pharmacy, The Affiliated Hospital of Chengde Medical College, Chengde, China \\ Email: ^hujhcyfy@163.com
}

How to cite this paper: Zhao, Y.W., Hu, J.H., Song, J., Zhao, X.H., Shi, Y.J. and Jiang, Y.P. (2020) Exploration on Shufeng Jiedu Capsule for Treatment of COVID-19 Based on Network Pharmacology and Molecular Docking. Chinese Medicine, 11, 9-18.

https://doi.org/10.4236/cm.2020.111002

Received: February 3, 2020

Accepted: March 28, 2020

Published: March 31, 2020

Copyright $\odot 2020$ by author(s) and Scientific Research Publishing Inc. This work is licensed under the Creative Commons Attribution International License (CC BY 4.0).

http://creativecommons.org/licenses/by/4.0/ (c) (i) Open Access

\begin{abstract}
The paper is proposed to explore the potential effects of Shufeng Jiedu Capsule against COVID-19. The ingredients and targets of Shufeng Jiedu Capsule were collected by the Traditional Chinese Medicine Systems Pharmacology Database and Analysis Platform (TCMSP), and the gene names of potential targets were extracted by UniProtKB. Then we did protein-protein interaction networks functional enrichment analysis by the STRING platform, reconstructed drug-target pathways and networks to predict the likely protein targets of the capsule against COVID-19 with software Cytoscape 3.6.1, and carried out GO enrichment analysis and KEGG analysis with R 5.3.2 software. At last we validated our predictions on molecular docking. The results suggested that Shufeng Jiedu Capsule contained 155 ingredients and 237 targets, including 26 main active ingredients and 45 key targets. There were 2334 biological processes (BP), 103 cell composition (CC) and 198 molecular functions (MF) in GO Enrichment Analysis, and 177 pathways in the KEGG analysis. The molecular docking analysis showed that binding energy for 26 main active ingredients ranged from -32.21 to $-25.94 \mathrm{~kJ} \cdot \mathrm{mol}^{-1}$, and the main targets bind to SARS-CoV-2 3CL hydrolase by acting on CASP9, PRKCA, RELA and others. Our study suggested that Shufeng Jiedu Capsule has potential therapeutic effects on COVID-19.
\end{abstract}

\section{Keywords}

Shufeng Jiedu Capsule, Network Pharmacology, Molecular Docking, COVID-19

\section{Introduction}

Coronavirus disease 2019 (COVID-19) is caused by a novel coronavirus and is highly infectious. It was named by the World Health Organization on January 12, 2020, and its pathogen was named SARS-CoV-2 [1]. From the traditional 
Chinese medicine angle, COVID-19 is a blight [2] [3]. Chinese medicine therapy is recommended from the third edition of "Novel Coronavirus Pneumonia Diagnosis and Treatment Scheme" issued by National Health Commission of the People's Republic of Chian and National Administration of Traditional Chinese Medicine, and achieved good results in treatment [4] [5] [6] [7]. Shufeng Jiedu Capsule was recommended from the sixth edition of "Novel Coronavirus Pneumonia Diagnosis and Treatment Scheme"; the formula was from "Qudu San", which is made from Polygoni Cuspidati Rhizoma Et Radix (Huzhang), Forsythiae Fructus (Lianqiao), Isatidis Radix (Banlangen), Radix Bupleuri (Chaihu), Herba Patriniae (Baijiangcao), Verbenae Herb (Mabiancao), Phragmitis Rhizoma (Lugen), and licorice (Gancao). The capsule has the functions of expelling wind, clearing heat and detoxification. Clinical studies showed that the capsule had antibacterial, antiviral and immunomodulatory effects, and had a good effect in the treatment of acute upper respiratory tract infection and community-acquired pneumonia [8] [9] [10] [11]. In this study, we selected relevant targets and pathological mechanisms of Shufeng Jiedu Capsule for the treatment of COVID-19 by network pharmacology and molecular docking [12], and then provided the basis for further study.

\section{Materials and Methods}

\subsection{The Main Active Components and Corresponding Targets of Shufeng Jiedu Capsule}

Searched names of 8 the traditional Chinese medicine (TCM) in Shufeng Jiedu Capsule as Polygoni Cuspidati Rhizoma Et Radix (Huzhang), Forsythiae Fructus (Lianqiao), Isatidis Radix (Banlangen), Radix Bupleuri (Chaihu), Herba Patriniae (Baijiangcao), Verbenae Herb (Mabiancao), Phragmitis Rhizoma (Lugen), licorice (Gancao), selected the active components and corresponding targets with filter conditions as oral bioavailability $(\mathrm{OB}) \geq 30$, drug-like $(\mathrm{DL}) \geq 0.18$.

\subsection{Constructed Drug-Compound-Target Network}

The gene symbols of the active components of Shufeng Jiedu Capsules were extracted from UniProtKB (http://www.uniprot.org), and the target was limited to Homo sapiens. Then construct PPI network with filter conditions as median confidence $>0.4$. Then construct drug-compound-target network by software Cytoscape 3.6.1, then select potential active components of Shufeng Jiedu Capsule against COVID-19 according to the node degree method [13].

\subsection{Gene Ontology Analysis and Pathway Analysis}

The targets of active components of Shufeng Jiedu Capsule were analyzed by Gene Ontology (GO) analysis and Kyoto Encyclopedia of Genes and Genomes (KEGG) pathway analysis. GO analysis included Biological Process (BP), Molecular Function (MF), Cellular Component (CC). Two analyses were worked on software R 5.3.2 with package of “org.Hs.eg.db", "clusterProfiler", "enrichplot" 
and "ggplot2" [14], adjust $\mathrm{P}<0.05$.

\subsection{Molecular Docking}

Download the active ingredients of Shufeng Jiedu Capsule from TCMSP and saved them in mol2 format, then saved them in PDBQT format through software Auto Dock. The 3D structure (PDB ID: 6LU7) of SARS-CoV-2 3CL hydrolase and angiotensin converting enzyme II (ACE2) download from RCSB database (http://www.rcsb.org/), then saved in PDB format, removed water and other small molecules, added hydrogenation through software Auto Dock, then saved in PDBQT format. Made molecular docking by software Vina (http://vina.scripps.edu/index.html), then selected the minimum binding energy of drug and protein as the optimal site, viewed and saved images by software Discovery Studio 2016.

\section{Results}

\subsection{The Main Active Components and Corresponding Targets of Shufeng Jiedu Capsule}

Selected 207 components of Shufeng Jiedu Capsule through TCMSP platform, included 10 of Polygoni Cuspidati Rhizoma Et Radix (Huzhang), 23 of Forsythiae Fructus (Lianqiao), 39 of Isatidis Radix (Banlangen), 17 of Radix Bupleuri (Chaihu), 13 of Herba Patriniae (Baijiangcao), 12 of Verbenae Herb (Mabiancao), 1 of Phragmitis Rhizoma (Lugen), 92 of Phragmitis Rhizoma (Gancao).

\subsection{Constructed Drug-Compound-Target Network}

Drug-compound-target network of Shufeng Jiedu Capsule includes 400 nodes, 7756 edges, 8 TCM, 155 active compounds, 236 targets. As shown in Figure 1, green-TCM, yellow-compounds, red-targets. Our study also selected key nods according to degree of topological analysis, including 3 TCM, 45 targets, 26 compounds (shown in Table 1).

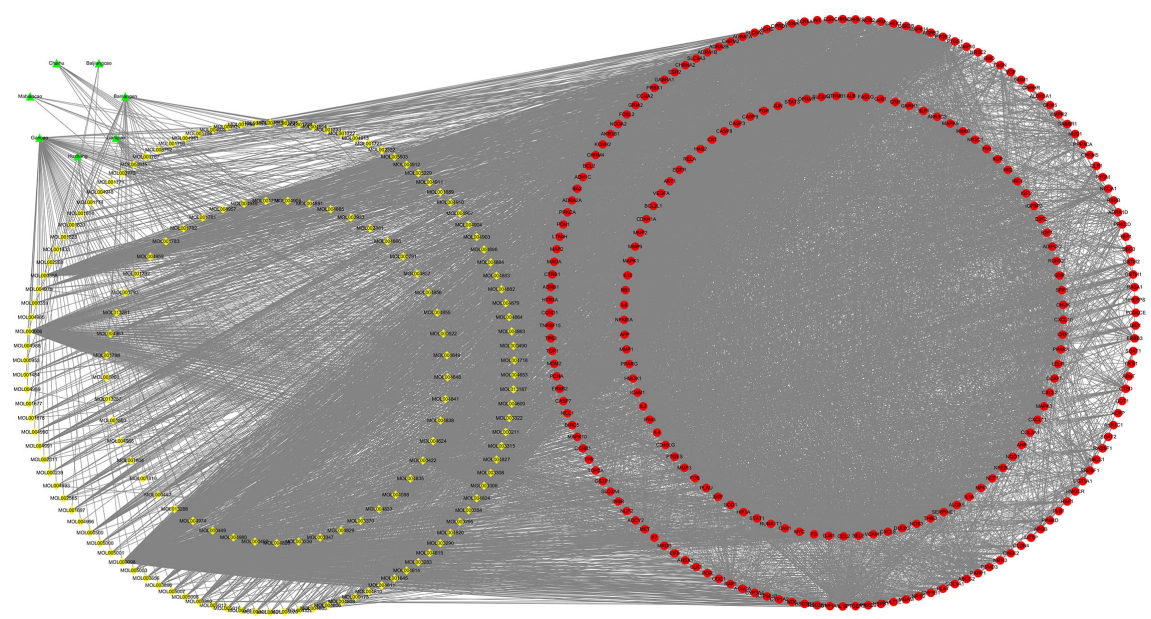

Figure 1. Drug-compound-target network of Shufeng Jiedu Capsule. 
Table 1. Main active components of Shufeng Jiedu Capsule.

\begin{tabular}{|c|c|c|c|c|c|}
\hline & Mol ID & Components & MW & OB (\%) & DL \\
\hline \multirow{4}{*}{ Huzhang } & MOL013287 & Physovenine & 262.34 & 106.21 & 0.19 \\
\hline & MOL000358 & beta-sitosterol & 414.79 & 36.91 & 0.75 \\
\hline & MOL000006 & luteolin & 286.25 & 36.16 & 0.25 \\
\hline & MOL000098 & quercetin & 302.25 & 46.43 & 0.28 \\
\hline \multirow{6}{*}{ Lianqiao } & MOL000173 & wogonin & 284.28 & 30.68 & 0.23 \\
\hline & MOL000358 & beta-sitosterol & 414.79 & 36.91 & 0.75 \\
\hline & MOL000422 & kaempferol & 286.25 & 41.88 & 0.24 \\
\hline & MOL000006 & luteolin & 286.25 & 36.16 & 0.25 \\
\hline & MOL000791 & bicuculline & 367.38 & 69.67 & 0.88 \\
\hline & MOL000098 & quercetin & 302.25 & 46.43 & 0.28 \\
\hline \multirow{3}{*}{ Banlangen } & MOL001689 & acacetin & 284.28 & 34.97 & 0.24 \\
\hline & MOL000358 & beta-sitosterol & 414.79 & 36.91 & 0.75 \\
\hline & MOL000449 & Stigmasterol & 412.77 & 43.83 & 0.76 \\
\hline \multirow{6}{*}{ Baijiangcao } & MOL001689 & acacetin & 284.28 & 34.97 & 0.24 \\
\hline & MOL000358 & beta-sitosterol & 414.79 & 36.91 & 0.75 \\
\hline & MOL000422 & kaempferol & 286.25 & 41.88 & 0.24 \\
\hline & MOL000449 & Stigmasterol & 412.77 & 43.83 & 0.76 \\
\hline & MOL000006 & luteolin & 286.25 & 36.16 & 0.25 \\
\hline & MOL000098 & quercetin & 302.25 & 46.43 & 0.28 \\
\hline \multirow{4}{*}{ Chaihu } & MOL000449 & Stigmasterol & 412.77 & 43.83 & 0.76 \\
\hline & MOL000354 & isorhamnetin & 316.28 & 49.6 & 0.31 \\
\hline & MOL000422 & kaempferol & 286.25 & 41.88 & 0.24 \\
\hline & MOL000098 & quercetin & 302.25 & 46.43 & 0.28 \\
\hline \multirow{6}{*}{ Mabiancao } & MOL000358 & beta-sitosterol & 414.79 & 36.91 & 0.75 \\
\hline & MOL000422 & kaempferol & 286.25 & 41.88 & 0.24 \\
\hline & MOL000449 & Stigmasterol & 412.77 & 43.83 & 0.76 \\
\hline & MOL000006 & luteolin & 286.25 & 36.16 & 0.25 \\
\hline & MOL002773 & beta-carotene & 536.96 & 37.18 & 0.58 \\
\hline & MOL000098 & quercetin & 302.25 & 46.43 & 0.28 \\
\hline \multirow[t]{3}{*}{ Lugen } & MOL000449 & Stigmasterol & 412.77 & 43.83 & 0.76 \\
\hline & MOL002565 & Medicarpin & 270.3 & 49.22 & 0.34 \\
\hline & MOL000354 & isorhamnetin & 316.28 & 49.6 & 0.31 \\
\hline \multirow[t]{3}{*}{ Gancao } & MOL003896 & 7-Methoxy-2-methyl isoflavone & 266.31 & 42.56 & 0.2 \\
\hline & MOL000392 & formononetin & 268.28 & 69.67 & 0.21 \\
\hline & MOL000422 & kaempferol & 286.25 & 41.88 & 0.24 \\
\hline
\end{tabular}




\begin{tabular}{lllcc}
\hline MOL004328 & naringenin & 272.27 & 59.29 & 0.21 \\
MOL004835 & Glypallichalcone & 284.33 & 61.6 & 0.19 \\
MOL004891 & shinpterocarpin & 322.38 & 80.3 & 0.73 \\
MOL004908 & Glabridin & 324.4 & 53.25 & 0.47 \\
MOL004957 & HMO & 268.28 & 38.37 & 0.21 \\
MOL004959 & 1-Methoxyphaseollidin & 354.43 & 69.98 & 0.64 \\
MOL004966 & 3'-Hydroxy-4'-O-Methylglabridin & 354.43 & 43.71 & 0.57 \\
MOL000497 & licochalcone a & 338.43 & 40.79 & 0.29 \\
MOL004974 & 3'-Methoxyglabridin & 354.43 & 46.16 & 0.57 \\
MOL004978 & 2-[(3R)-8,8-dimethyl-3,4-dihydro-2H-pyrano[6,5-f]chromen-3-yl]-5-methoxyphenol & 338.43 & 36.21 & 0.52 \\
MOL000500 & Vestitol & 272.32 & 74.66 & 0.21 \\
MOL005003 & Licoagrocarpin & 338.43 & 58.81 & 0.58 \\
MOL000098 & quercetin & 302.25 & 46.43 & 0.28 \\
\hline
\end{tabular}

\subsection{Gene Ontology Analysis and Pathway Analysis}

We got 2363 GO items through Gene ontology analysis, including 2334 BP items, 103 CC items, 198 MF items. We also got 177 pathways through KEGG analysis, including Human cytomegalovirus infection, PI3K-Akt signaling pathway and Hepatitis B, etc. The results are shown in Figure 2.

\subsection{Molecular Docking}

Molecular docking with SARS-CoV-2 3CL hydrolase was carried out between the 26 main active components of Shufeng Jiedu Capsule selected above. The lower the binding energy, the greater the possibility of interaction between the active compound and the hydrolase. The binding energy of 26 compounds is from -32.21 to $-20.94 \mathrm{~kJ} \cdot \mathrm{mol}^{-1}$ (shown in Table 2). Bicuculline (MOL000791) had the greatest effect with hydrolase $\left(-32.21 \mathrm{~kJ} \cdot \mathrm{mol}^{-1}\right)$, which is the active compound of Forsythiae Fructus (Lianqiao), the second was MOL004978 $\left(-30.54 \mathrm{~kJ} \cdot \mathrm{mol}^{-1}\right)$, the third was MOL004966 $\left(-30.12 \mathrm{~kJ} \cdot \mathrm{mol}^{-1}\right)$, the results are shown in Figure 3.

\section{Discussion}

Currently, there is no specific drug treatment for COVID-19, and existing chemical drugs can only inhibit some clinical symptoms within a limited range, but within higher cure rate and lower mortality rate of confirmed patients with TCM treatment [15]. The sixth edition of "Novel Coronavirus Pneumonia Diagnosis and Treatment Scheme" advised different TCM treatments according to TCM dialectics in treatment in patients in different pathological stages, and Shufeng Jiedu Capsule was recommended for prevention and treatment during medical observation stage. 
Table 2. The binding energy of 26 compounds and SARS-CoV-2 3CL hydrolase.

\begin{tabular}{|c|c|c|c|c|c|c|c|c|c|c|}
\hline \multirow{2}{*}{ MOL ID } & \multirow{2}{*}{ Compound } & \multicolumn{8}{|c|}{ Shufeng Jiedu Capsule } & \multirow{2}{*}{$\begin{array}{l}\text { binding } \\
\text {-energy } \\
\left(\mathrm{kJ} \cdot \mathrm{mol}^{-1}\right)\end{array}$} \\
\hline & & $\mathrm{HZ}$ & LQ & BLG & $\mathrm{BJC}$ & $\mathrm{CH}$ & $\mathrm{MBC}$ & LG & GC & \\
\hline MOL000098 & quercetin & $\sqrt{ }$ & $\sqrt{ }$ & - & $\sqrt{ }$ & $\sqrt{ }$ & $\sqrt{ }$ & - & $\sqrt{ }$ & -25.52 \\
\hline MOL000422 & kaempferol & - & $\sqrt{ }$ & - & $\sqrt{ }$ & $\sqrt{ }$ & $\sqrt{ }$ & - & $\sqrt{ }$ & -25.10 \\
\hline MOL000006 & luteolin & $\sqrt{ }$ & $\sqrt{ }$ & - & $\sqrt{ }$ & - & $\sqrt{ }$ & - & - & -28.45 \\
\hline MOL000358 & beta-sitosterol & $\sqrt{ }$ & $\sqrt{ }$ & $\sqrt{ }$ & $\sqrt{ }$ & - & $\sqrt{ }$ & - & - & -25.94 \\
\hline MOL000449 & Stigmasterol & - & - & $\sqrt{ }$ & $\sqrt{ }$ & $\sqrt{ }$ & $\sqrt{ }$ & $\sqrt{ }$ & - & -26.35 \\
\hline MOL000354 & isorhamnetin & - & - & - & - & $\sqrt{ }$ & - & - & $\sqrt{ }$ & -24.68 \\
\hline MOL000173 & wogonin & - & $\sqrt{ }$ & - & - & - & - & - & - & -25.94 \\
\hline MOL001689 & acacetin & - & - & $\sqrt{ }$ & $\sqrt{ }$ & - & - & - & - & -24.68 \\
\hline MOL003896 & 7-Methoxy-2-methyl isoflavone & - & - & - & - & - & - & - & $\sqrt{ }$ & -23.84 \\
\hline MOL004328 & naringenin & - & - & - & - & - & - & - & $\sqrt{ }$ & -26.35 \\
\hline MOL000392 & formononetin & - & - & - & - & - & - & - & $\sqrt{ }$ & -28.86 \\
\hline MOL013287 & Physovenine & $\sqrt{ }$ & - & - & - & - & - & - & - & -25.10 \\
\hline MOL000497 & Licochalcone A & - & - & - & - & - & - & - & $\sqrt{ }$ & -25.94 \\
\hline MOL004891 & Shinpterocarpin & - & - & - & - & - & - & - & $\sqrt{ }$ & -28.45 \\
\hline MOL004978 & $\begin{array}{l}\text { 2-[(3R)-8,8-dimethyl-3,4-dihydro-2H-pyrano[6,5-f] } \\
\text { chromen-3-yl]-5-methoxyphenol }\end{array}$ & - & - & - & - & - & - & - & $\sqrt{ }$ & -30.54 \\
\hline MOL002565 & Medicarpin & - & - & - & - & - & - & - & $\sqrt{ }$ & -25.94 \\
\hline MOL000500 & Vestitol & - & - & - & - & - & - & - & $\sqrt{ }$ & -24.26 \\
\hline MOL005003 & Licoagrocarpin & - & - & - & - & - & - & - & $\sqrt{ }$ & -27.61 \\
\hline MOL002773 & beta-carotene & - & - & - & - & - & $\sqrt{ }$ & - & - & -28.45 \\
\hline MOL004835 & Glypallichalcone & - & - & - & - & - & - & - & $\sqrt{ }$ & -22.17 \\
\hline MOL004974 & 3'-Methoxyglabridin & - & - & - & - & - & - & - & $\sqrt{ }$ & -29.70 \\
\hline MOL000791 & bicuculline & - & $\sqrt{ }$ & - & - & - & - & - & - & -32.21 \\
\hline MOL004908 & Glabridin & - & - & - & - & - & - & - & $\sqrt{ }$ & -28.45 \\
\hline MOL004957 & $\mathrm{HMO}$ & - & - & - & - & - & - & - & $\sqrt{ }$ & -20.92 \\
\hline MOL004959 & 1-Methoxyphaseollidin & - & - & - & - & - & - & - & $\sqrt{ }$ & -24.26 \\
\hline MOL004966 & 3'-Hydroxy-4'-O-Methylglabridin & - & - & - & - & - & - & - & $\sqrt{ }$ & -30.12 \\
\hline
\end{tabular}

Note: 1) HZ-Polygoni Cuspidati Rhizoma Et Radix, LQ-Forsythiae Fructus, Isatidis BLG-Radix, CH—Radix Bupleuri, BJCb-Herba Patriniae, MBC-Verbenae Herb, LG-Phragmitis Rhizoma, licorice-GC. 2) $\sqrt{ }$-contain compound, -- free from compound. 


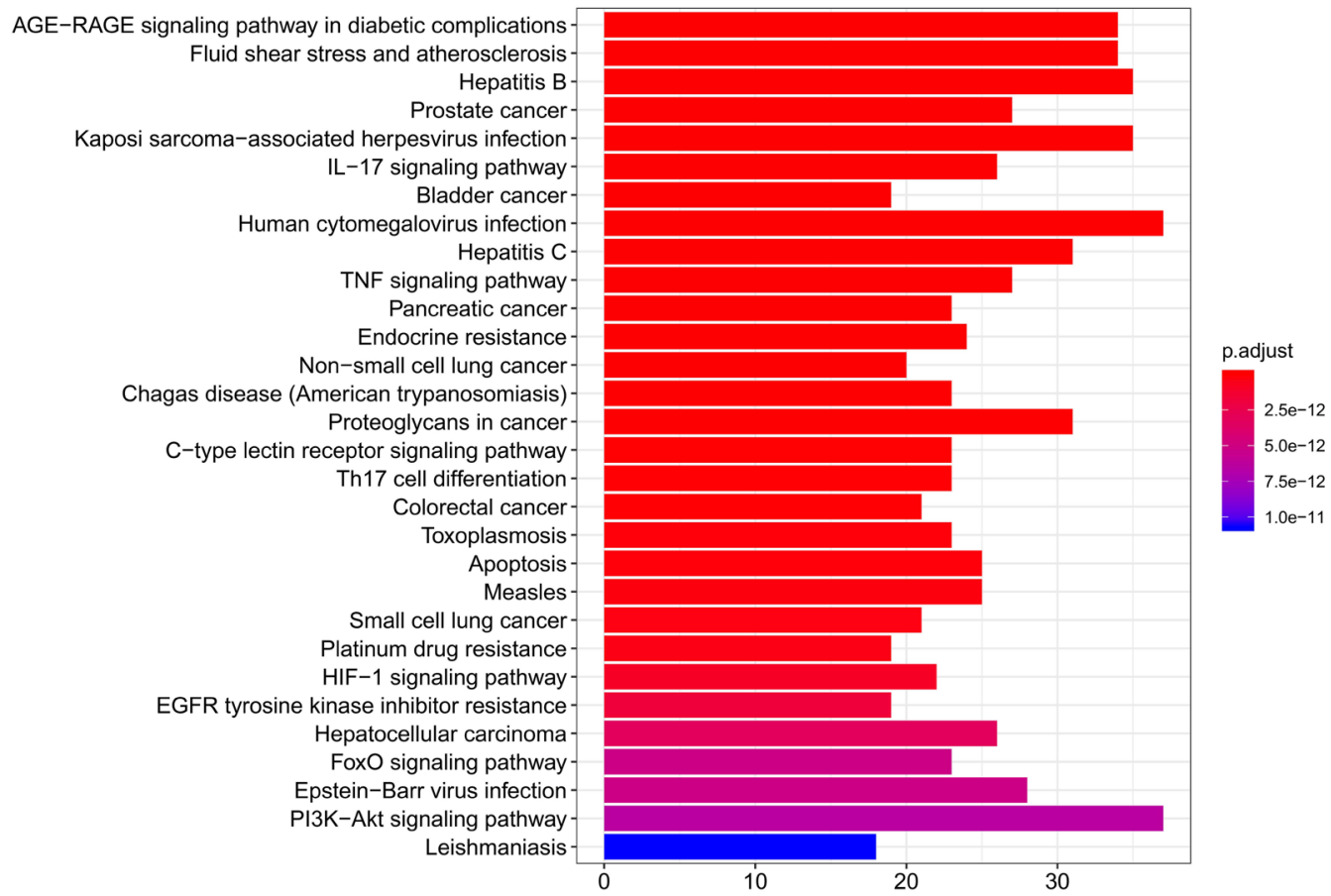

GO

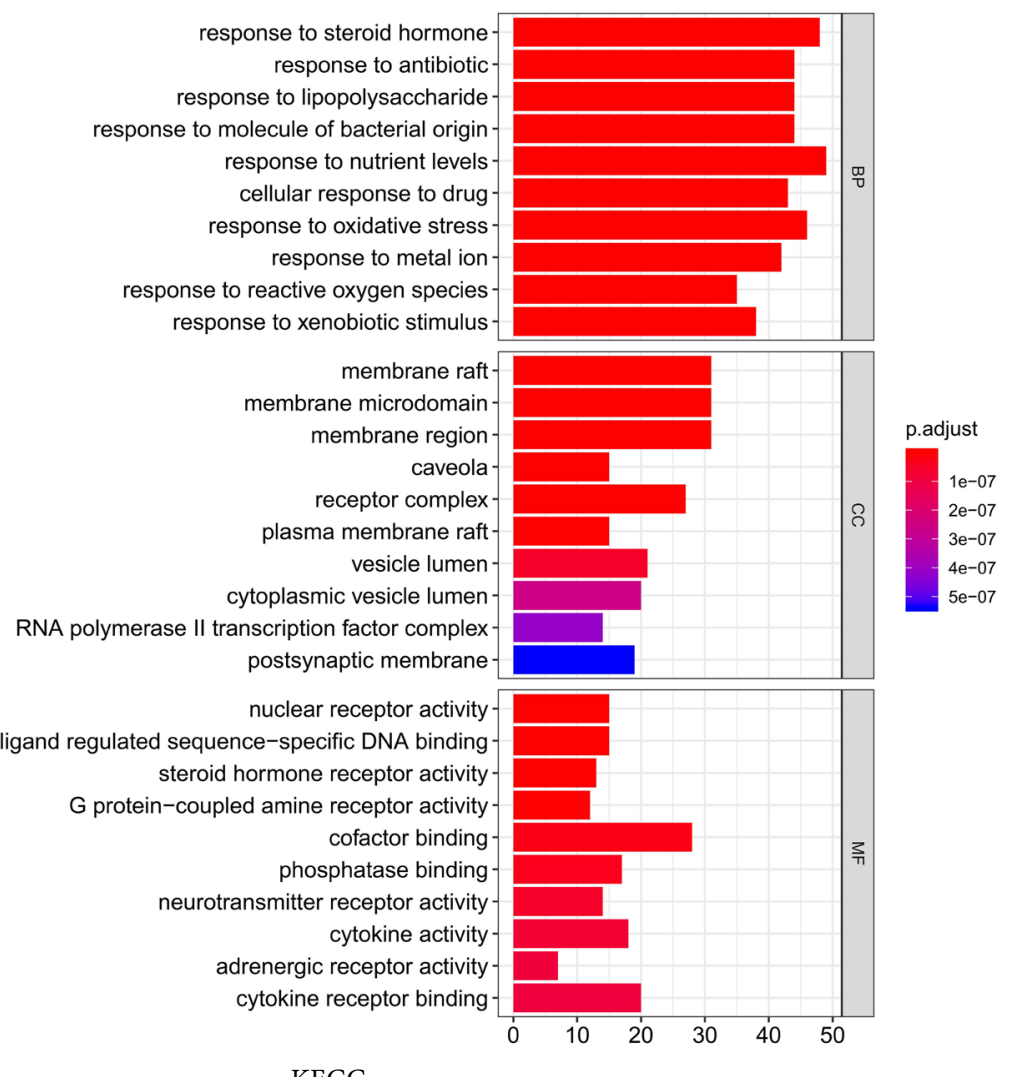

KEGG

Figure 2. Gene ontology analysis and KEGG pathway analysis of Shufeng Jiedu Capsule. (The picture above is gene ontology analysis, the picture below is KEGG pathway analysis. In the figure, the gradient from blue to red represents that the smaller the $\mathrm{P}$ value is, and the gradient from small to large represents the larger the number of enrichment targets.) 

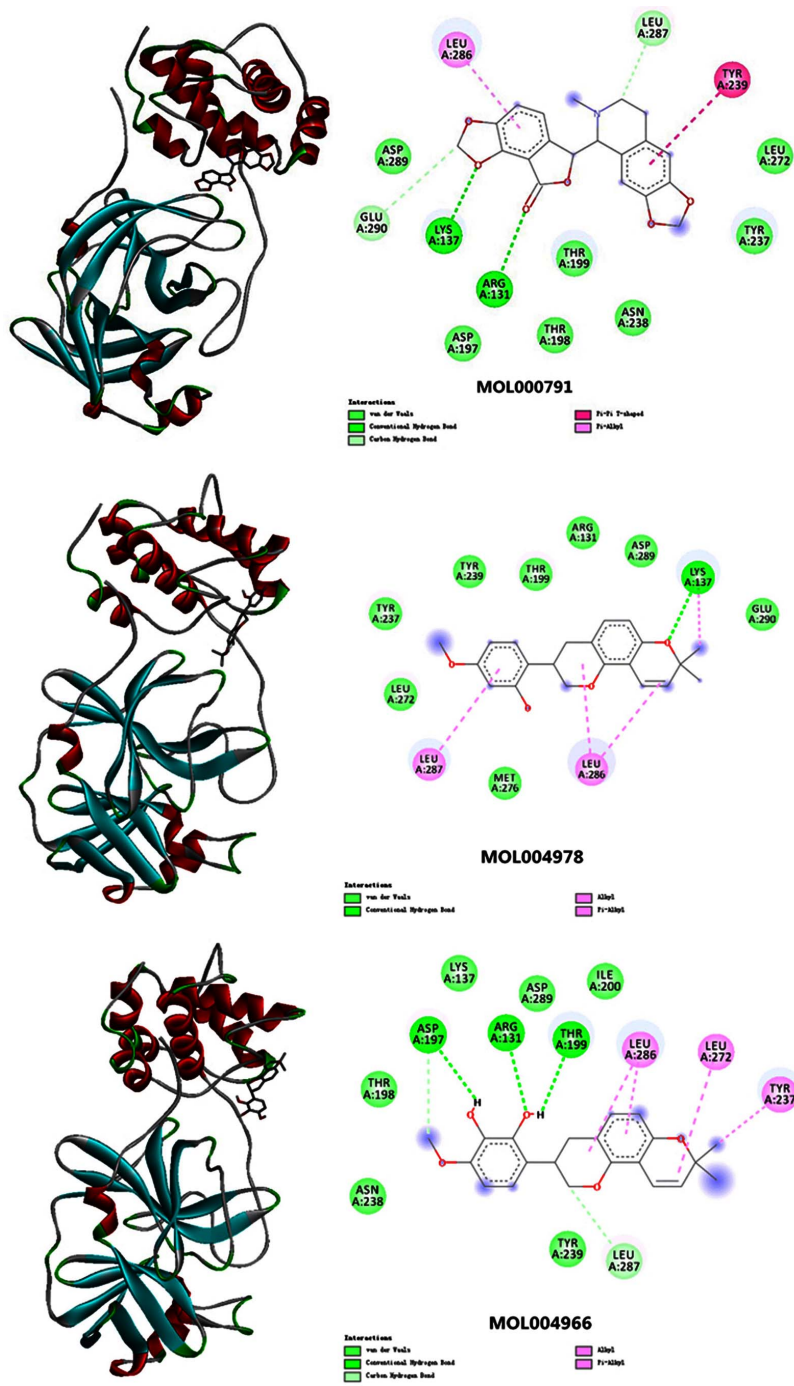

Figure 3. Molecular docking mode of 3 active compounds in Shufeng Jiedu Capsule and SARS-CoV-2 3CL hydrolase.

In this study, through network pharmacology and molecular docking, we got 26 active compounds of Shufeng Jiedu Capsule, and binding energy of compounds and SARS-CoV-2 3CL hydrolase were from -32.21 to $-20.94 \mathrm{~kJ} \cdot \mathrm{mol}^{-1}$. Quercetin $\left(-25.52 \mathrm{~kJ} \cdot \mathrm{mol}^{-1}\right)$ in our study is similar to the published research [13], whose binding energy with SARS-CoV-2 3CL hydrolase was -26.36 $\mathrm{kJ} \cdot \mathrm{mol}^{-1}$. The results of molecular docking are shown that combination of Shufeng Jiedu Capsule and hydrolase includes hydrogen bond, pi-pi conjugate and van der Waals force. Moreover, the capsule contains many active compounds, then it can be predicted that Shufeng Jiedu Capsule has a high possibility of anti-COVID-19.

Our study selected the main compounds and targets of Shufeng Jiedu Capsule by network pharmacology, then analyzed the potential molecular mechanism on its binding energy with SARS-CoV-2 3CL hydrolase by molecular docking. The results showed that Shufeng Jiedu Capsule acts on hydrolase by multiple com- 
pounds, targets and pathways. The method in this study provides the basis in mechanism of action of Chinese herbal compound preparation. However, this study still has some limitations, such as insufficient understanding of the pathogenesis of COVID-19 and insufficient research on the key targets of the viral infection process. In this study, the pharmacodynamics, evaluation and clinical efficacy of COVID-19 were studied and discussed at a theoretical level. In the later stage, the pharmacodynamics, evaluation and clinical efficacy should be further studied to provide a basis for further development and utilization of COVID-19 therapeutic drugs.

\section{Funding}

This study was funded by the Study and Development Fund for Sciences and Technology in Chengde City (No. 201701A087).

\section{Conflicts of Interest}

The authors declare no conflicts of interest regarding the publication of this paper.

\section{References}

[1] Sohrabi, C., Alsafi, Z., O’Neill, N., et al. (2020) World Health Organization Declares Global Emergency: A Review of the 2019 Novel Coronavirus (COVID-19) [published online ahead of print, 2020 Feb 26]. International Journal of Surgery, 77, 217. https://doi.org/10.1016/j.ijsu.2020.03.036

[2] Ma, J.J., Chen, M. and Wang, Y.G. (2020) Novel Coronavirus (2019-NCOV) Syndrome: A Review of TCM Syndrome Treatment. Beijing Journal of Traditional Chinese Medicine, 39, 95-101.

[3] Sun, Z.T., An, X., Xiao, W., et al. (2020) Discussion on Differentiation and Treatment of COVID-19 by Stages. Journal of Shaanxi University of Chinese Medicine. http://kns.cnki.net/kcms/detail/61.1501.R.20200211.1318.002.html

[4] http://www.gov.cn/zhengce/zhengceku/2020-01/23/content_5471832.htm

[5] http://www.gov.cn/zhengce/zhengceku/2020-01/28/content_5472673.htm

[6] http://www.gov.cn/zhengce/zhengceku/2020-02/05/content_5474791.htm

[7] http://www.gov.cn/zhengce/zhengceku/2020-02/19/content_5480948.htm

[8] Lv, W.W., Zhu, T.N., Qiu, H., et al. (2013) Pharmacodynamic Study on Antiviral and Antibacterial Effects of Shufeng Jiedu Capsules in Vitro. Traditional Chinese Drug Research and Clinical Pharmacology, 24, 234-238.

[9] Xu, Y.L., Xue, Y.L., Zhang, H.H., et al. (2015) Clinical Observation on Treatment of Acute Upper Respiratory Infection of Wind-Heat Syndrome with Shufeng Jiedu Capsules: A Randomize-Controlled Double-Blind Test. Journal of Traditional Chinese Medicine, 56, 676-679.

[10] Liu, R., Li, X.L., Wang, L.Y., et al. (2018) Meta-Analysis and GRADE Assessment of Shufeng Jiedu Capsule Combined with Antibiotics in Treating Community Acquired Pneumonia. Journal of Traditional Chinese Medicine, 59, 1656-1660.

[11] Zhang, B. and Zhang, W. (2020) Clinical Effect of Shufeng Jiedu Capsules for Acute Upper Respiratory Tract Infection and Its Effect on Inflammatory Factors. Journal 
of New Chinese Medicine, 52, 50-53.

[12] Xiang, Y. and Lv, W.L. (2020) Study on the Mechanism of Xue's Wuye Lugen Decoction Tea Drink on the Prevention and Control of COVID-19 Based on Network Pharmacology and Molecular Docking. Journal of Hubei University of Chinese Medicine. http://kns.cnki.net/kcms/detail/42.1844.R.20200303.1940.002.html

[13] Yao, Y.X., He, Z.X., Liu, X.F., et al. (2019) Potential Material Basis of Kangbingdu Granules for Treatment of Coronavirus Disease 2019 (COVID-19) Based on Network Pharmacology and Molecular Docking Technology. Chinese Traditional and Herbal Drugs, 51, 1386-1396.

[14] Yu, G., Wang, L.G., Han, Y., et al. (2012) clusterProfiler: An R Package for Comparing Biological Themes among Gene Clusters. OMICS, 16, 284-287. https://doi.org/10.1089/omi.2011.0118

[15] Chen, J., Wang, W.Q., Shi, C.Y., et al. (2020) Thoughts on Prevention and Treatment of Coronavirus Disease 2019 (COVID-19) by Traditional Chinese Medicine. Chinese Traditional and Herbal Drugs, 51, 1106-1112. 